\title{
Sera from patients with active systemic lupus erythematosus patients enhance the toll-like receptor 4 response in monocyte subsets
}

Tiago Carvalheiro', Diane Gomes², Ligia A. Pinto ${ }^{3}$, Luis Inês ${ }^{4,5,6}$, Ana Lopes ${ }^{1}$, Ana Henriques ${ }^{5}$, Susana Pedreiro ${ }^{1}$, António Martinho ${ }^{1}$, Hélder Trindade ${ }^{1}$, Howard A. Young ${ }^{7}$, José António Pereira da Silva ${ }^{4,5}$ and Artur Paiva ${ }^{1,2^{*}}$

\begin{abstract}
Background: Systemic Lupus Erythematosus (SLE) is an auto-immune disease whose complex pathogenesis remains unraveled. Here we aim to explore the inflammatory ability of SLE patients' sera upon peripheral blood (PB) monocyte subsets and myeloid dendritic cells (mDCs) obtained from healthy donors.

Methods: In this study we included 11 SLE patients with active disease (ASLE), 11 with inactive disease (ISLE) and 10 healthy controls (HC). PB from healthy donors was stimulated with patients' sera, toll-like receptor (TLR) 4 ligand lipopolysaccharide or both. The intracellular production of TNF-a was evaluated in classical, non-classical monocytes and mDCs, using flow cytometry. TNF-a mRNA expression was assessed in all these purified cells, after sera treatment.

Results: We found that sera of SLE patients did not change spontaneous TNF-a production by monocytes or dendritic cells. However, upon stimulation of TLR4, the presence of sera from ASLE patients, but not ISLE, significantly increased the intracellular expression of TNF-a in classical and non-classical monocytes. This ability was related to titers anti-double stranded DNA antibodies in the serum. High levels of anti-TNF-a in the patients' sera were associated with increased TNF-a expression by co-cultured mDCs. No relationship was found with the levels of a wide variety of other pro-inflammatory cytokines. A slight increase of TNF-a mRNA expression was observed in these purified cells when they were cultured only in the presence of SLE serum.
\end{abstract}

Conclusions: Our data suggest that SLE sera induce an abnormal in vitro TLR4 response in classical and non-classical monocytes, reflected by a higher TNF-a intracellular expression. These effects may be operative in the pathogenesis of SLE.

Keywords: Systemic lupus erythematosus, Serum, Cytokines, Toll like receptor 4, Classical monocytes, Non-classical monocytes, Myeloid dendritic cells

\section{Background}

Systemic lupus erythematosus (SLE) is a systemic autoimmune disease characterized by heterogeneous clinical manifestations with varying degrees of severity and alternating phases of remission and flare. The primary pathological findings in SLE patients are inflammation, vasculitis, immune complex deposition, and vasculopathy

\footnotetext{
* Correspondence: artur.paiva@ipst.min-saude.pt

'Blood and Transplantation Center of Coimbra, Portuguese Institute of Blood and Transplantation, Quinta da Vinha Moura, São Martinho do Bispo,

3041-861 Coimbra, Portugal

${ }^{2}$ College of Health Technology of Coimbra, Rua 5 de Outubro, São Martinho do Bispo, 3046-854 Coimbra, Portugal

Full list of author information is available at the end of the article
}

[1-4]. The disease is characterized by the presence of a variety of autoantibodies against cell components and circulating proteins, which are associated with differing disease manifestations [5].

Monocytes are key players in both innate and adaptive immune responses since they can produce large amounts of soluble cytokines and are equipped with a large array of receptors proving them with the ability of recognizing lipids, dying cells, microorganisms and their derivates. Given the plasticity of monocytes and their distinct role in inflammation, repair and healing processes $[6,7]$, according to the recommendations of Ziegler-Heitbrock et al. it is possible to subdivide human monocytes into three 
subsets on the basis of the expression of CD14 and the CD16 receptors. The classical monocytes show high CD14 expression but no CD16 (CD14++CD16-), the intermediate monocytes show a high level of CD14 together with low CD16 (CD14++CD16+), and the nonclassical monocytes express a low level of CD14 together with high CD16 (CD14+CD16++) [8]. Functionally, classical monocytes are professional phagocytes that ingest native low-density lipoprotein, generate reactive oxygen species and secret cytokines in response to lipopolysaccharide (LPS) [9]. In contrast, non-classical monocytes do not generate reactive oxygen species and are weak phagocytes, taking-up preferentially oxidized low-density lipoprotein. They secrete substantial amounts of proinflammatory cytokines (tumor necrosis factor [TNF]$\alpha$, interleukin [IL]-1 $\beta$ and CCL3) after toll-like receptor (TLR) - dependent activation by viruses and nucleic acids [9-11].

It is also well accepted that dendritic cells (DCs) represent a heterogeneous population of potent lineage-negative HLA-DR+ antigen-presenting cells [12]. It is possible to identify at least two subsets of circulating DCs on the blood [8]; the myeloid $D C(m D C)$ subpopulation, strongly expressing HLA-DR and the myeloid-associated antigens $\mathrm{CD} 11 \mathrm{c}$ and $\mathrm{CD} 33$ and the plasmacytoid $D C$ ( $p D C s)$ subset, expressing high levels of both HLA-DR and CD123, without expressing myeloid associated antigens [13].

Abnormalities in monocytes and DCs from SLE patients have already been described [14-16] and an important role of DCs in SLE pathogenesis been advocated $[17,18]$. It has been reported that sera of SLE patients can induce the production of IFN- $\alpha$ in normal plasmacytoid DCs $[19,20]$. SLE sera have also been shown to induce the differentiation of normal monocytes into DCs [21], and to promote a B cell response [22]. This pro-inflammatory activity apparently requires the presence of circulating immune complexes in the sera [20]. However the ability of the soluble mediators presents in SLE patients' serum to activate normal peripheral blood monocytes subsets and mDCs is not well established.

In this context, we have evaluated the ability of sera from SLE patients to induce the intracellular production of TNF- $\alpha$ by classical, non-classical monocytes and mDCs. We examined the effect of SLE sera in the presence and absence of TLR4 stimulation by LPS. Interestingly, SLE sera did not change the intracellular production of TNF- $\alpha$ in the absence of TLR4 stimulation. However, the stimulation with TLR4 ligand in the presence of sera from ALSE resulted in a higher intracellular production of TNF- $\alpha$ by classical and non-classical monocytes, than in the presence of sera obtained from healthy controls and ISLE.

\section{Materials and methods Patients}

Twenty two patients with SLE, according to the 1997 American College of Rheumatology classification criteria [23] followed at the Lupus Clinic, Rheumatology Department of the University Hospital Center of Coimbra, were recruited and asked to provide a blood sample for this study. Disease activity was assessed at the time of blood collection, according to the SLE Disease Activity Index 2000 (SLEDAI 2 k) [23, 24]. SLE patients were divided into two groups, one with clinically active disease (ASLE) (SLEDAI $2 \mathrm{k} \geq 5 ; \mathrm{n}=11,100 \%$ female, median age 27: 19-52 year) and the other with clinically inactive disease (ISLE) (SLEDAI $2 \mathrm{k}<5 ; \mathrm{n}=11,64 \%$ female, median age 28: 19-45 year) [25]. Current medication details and additional routine laboratory parameters were collected from the patients' files (Table 1). Sera samples were stored at $-20{ }^{\circ} \mathrm{C}$ until analysis.

\section{Controls}

The healthy control group $(\mathrm{HC})$ comprised 10 healthy individuals ( $90.0 \%$ female, median age 28.5: 25 - 35 year)

Table 1 Clinical findings in 22 patients with systemic lupus erythematosus (SLE)

\begin{tabular}{|c|c|c|}
\hline & $\operatorname{ASLE}(n=11)$ & $\operatorname{ISLE}(n=11)$ \\
\hline Mean SLEDAI scores & $10.4 \pm 4.1$ & $1.5 \pm 1.2$ \\
\hline Mean time since diagnosis & $6.6 \pm 6.0$ & $8.3 \pm 4.9$ \\
\hline Lupus nephritis & $45.5 \%$ & $54.5 \%$ \\
\hline Neurolupus & $0 \%$ & $18.2 \%$ \\
\hline Lupus arthritis & $72.7 \%$ & $63.6 \%$ \\
\hline Hematological involvement & $100 \%$ & $90.9 \%$ \\
\hline Lupus cutaneous involvement & $72.7 \%$ & $72.7 \%$ \\
\hline Severe Lupus ${ }^{a}$ & $45.5 \%$ & $54.5 \%$ \\
\hline \multicolumn{3}{|l|}{ Anti-dsDNA antibodies ${ }^{b}$} \\
\hline Negative & $0 \%$ & $27.2 \%$ \\
\hline Low positive & $9.1 \%$ & $36.4 \%$ \\
\hline Moderate positive & $18.2 \%$ & $27.3 \%$ \\
\hline High positive & $72.7 \%$ & $9.1 \%$ \\
\hline \multicolumn{3}{|l|}{ Treatment } \\
\hline Hydroxychloroquine & $90.9 \%$ & $90.9 \%$ \\
\hline Immunossupressants $^{c}$ & $54.5 \%$ & $36.4 \%$ \\
\hline Steroids ${ }^{d}$ & $90.9 \%$ & $18.2 \%$ \\
\hline Low dose & $40.0 \%$ & $100 \%$ \\
\hline Moderate dose & $30.0 \%$ & $0 \%$ \\
\hline High dose & $30.0 \%$ & $0 \%$ \\
\hline
\end{tabular}

a Lupus severity in accordance with cumulative major organ involvement ${ }^{\mathrm{b}}$ Anti-dsDNA antibodies: low positive (<20 IU); moderate positive $(20-50 \mathrm{IU})$; high positive ( $>50 \mathrm{IU})$

cazathioprine, mycophenolate mofetil, cyclosporine, tacrolimus, methotrexate, cyclophosphamide or rituximab

'Low dose, up to $10 \mathrm{mg} /$ day; moderate dose, 10-30 mg/day; high dose, more than $30 \mathrm{mg} /$ day 
who provided blood samples for determination of serum cytokine levels. Five different healthy donors provided an additional blood samples ( $90 \%$ female; median age 29 : 25 - 33 years) collected in heparin anticoagulant tubes (6 mL of blood). These participants were required to complete a brief questionnaire regarding previous or current medical conditions and therapies. All were free from autoimmune disease, other active inflammatory conditions and medication with immunomodulatory drugs.

\section{Ethical aspects}

The study protocol was approved by the ethics committee of the University Hospital Center of Coimbra. All participants provided a signed informed consent prior to any study procedures and the principles of the Helsinki Declaration were fully respected.

\section{Peripheral blood cultures with sera from SLE patients and} HC with and without co-stimulation with TLR4 ligand - LPS To assess the effect of SLE sera upon TNF- $\alpha$ production by normal peripheral blood monocyte subsets and $\mathrm{mDCs}$, sera from 11 ASLE patients, 11 ISLE patients and 10 healthy individuals were used. The heparinized whole blood samples obtained from healthy donors were washed thrice with $\mathrm{NaCl} 0.9 \%$ saline solution and resuspended in $0.25 \mathrm{~mL}$; then samples were diluted (1:1) in RPMI-1640 medium (Life Technologies - Thermo Fisher Scientific; Carlsbad, C.A., USA), supplemented with $2 \mathrm{mM} \mathrm{L-glu-}$ tamine and antibiotic-antimycotic agent (Life Technologies - Thermo Fisher Scientific) in a total of $0.5 \mathrm{~mL}$. Four different conditions were created: 1) adding $0.05 \mathrm{~mL}$ of sera from patients with SLE or HC; 2) adding $100 \mathrm{ng} / \mathrm{mL}$ of LPS from Escherichia coli (serotype 055:B5; Sigma) plus $0.05 \mathrm{~mL}$ of sera from patients with SLE or HC; 3) adding $100 \mathrm{ng} / \mathrm{mL}$ of LPS from Escherichia coli (as a positive control) and 4) an unstimulated condition (as the negative control). All experiments included the presence of $10 \mu \mathrm{g} /$ mL of Brefeldin A (ref: B7651; Sigma, St. Louis, MO, USA) to prevent the release of cytokines from the cells. Samples were incubated for $6 \mathrm{~h}$ at $37{ }^{\circ} \mathrm{C}$ in a sterile environment with a $5 \% \mathrm{CO}_{2}$ humid atmosphere.

\section{Immunofluorescent staining}

After the $6 \mathrm{~h}$ incubation period, samples were aliquoted in two different tubes $(0.250 \mathrm{~mL} /$ tube $)$ in order to analyse the intracellular production of TNF- $\alpha$ in classical and non-classical monocytes as well as in mDCs. For the identification of these populations, cells were stained with the following monoclonal antibody combination: anti-CD45 krome orange (clone: J.33; Beckman Coulter - Immunotech, Marseille, France), anti-CD33 phycoerythrin cyanine 7 tandem (clone: D3HL60.251; Beckman Coulter - Immunotech) anti-CD14 allophycocyanin (clone: RM052; Beckman Coulter - Immunotech) and anti-HLA-DR peridinin chlorophyll protein cyanine 5 (clone: L243; Becton and Dickinson (BD) Biosciences, San Jose, CA, USA). After gentle mixing, cells were incubated for $15 \mathrm{~min}$ at room temperature in the dark followed by an intracytoplasmatic permeabilization protocol with IntraPrep Permeabilization Reagent (Beckman Coulter - Immunotech). Cells were fixed and permeabilized according to the manufacturer's instructions. Thereafter, anti-TNF- $\alpha$ antibody (clone MAb11; BD Pharmingen, San Diego, CA, USA) was added and incubated for $15 \mathrm{~min}$ at room temperature in the dark. The cells were then washed twice with phosphatebuffered saline (Gibco BRL-life Technologies) and resuspended in $0.250 \mathrm{~mL}$ of this buffer.

\section{Flow cytometry data acquisition and analysis}

Data acquisition was performed in a FACSCanto II flow cytometer (BD Biosciences) with the FACSDiva software (BD Biosciences) using the EuroFlow instrument setup data acquisition standard operating procedures [26]. For each sample at least 250.000 events were acquired.

Data analysis for each variable was performed using the flow cytometry software Infinicyt 1.6 (Cytognos, Salamanca, Spain). The evaluation of TNF- $\alpha$ production was based on the frequency (\%) of positive cells within each cell subset and their corresponding expression as determined by the mean fluorescence intensity (MFI), expressed as a relative logical scale.

Since CD16 expression is lost shortly after LPS stimulation, as also reported by others [27-30], thus precluding the identification of CD16+ monocyte subsets. On the other hand CD33 remains unchanged during LPS stimulation [30] and therefore CD33 was used as an alternative marker to CD16. Using a combination of anti-CD16 Pacific Blue (clone: 3G8; BD Pharmingen), anti-CD14 allophycocyanin, anti-HLA-DR peridinin chlorophyll protein cyanine 5 , anti-CD33 phycoerythrin cyanine 7 tandem and anti-CD45 krome orange in unstimulated cells it is possible distinguish between nonclassical and classical monocytes base on CD33 and CD14 combination (Fig. 1). mDCs were identified base on their CD $33^{\text {high }} /$ HLA-DR ${ }^{\text {high }}$ expression with intermediate forward and side scatter between lymphocytes and monocytes (Fig. 1) [15, 30].

TNF-a relative gene expression analysis after sorting of classical, non-classical monocytes, and mDCs, after culture in the presence of SLE or HC sera

We set out to evaluate the direct effect of sera from patients with SLE upon TNF- $\alpha$ mRNA expression by monocytes subsets and dendritic cells from normal individuals in the absence of LPS. For this purpose, a heparinized peripheral blood sample from one healthy subject was washed thrice with $\mathrm{NaCl} 0.9 \%$ saline solution and diluted 1:1 in RPMI-1640 medium supplemented with 


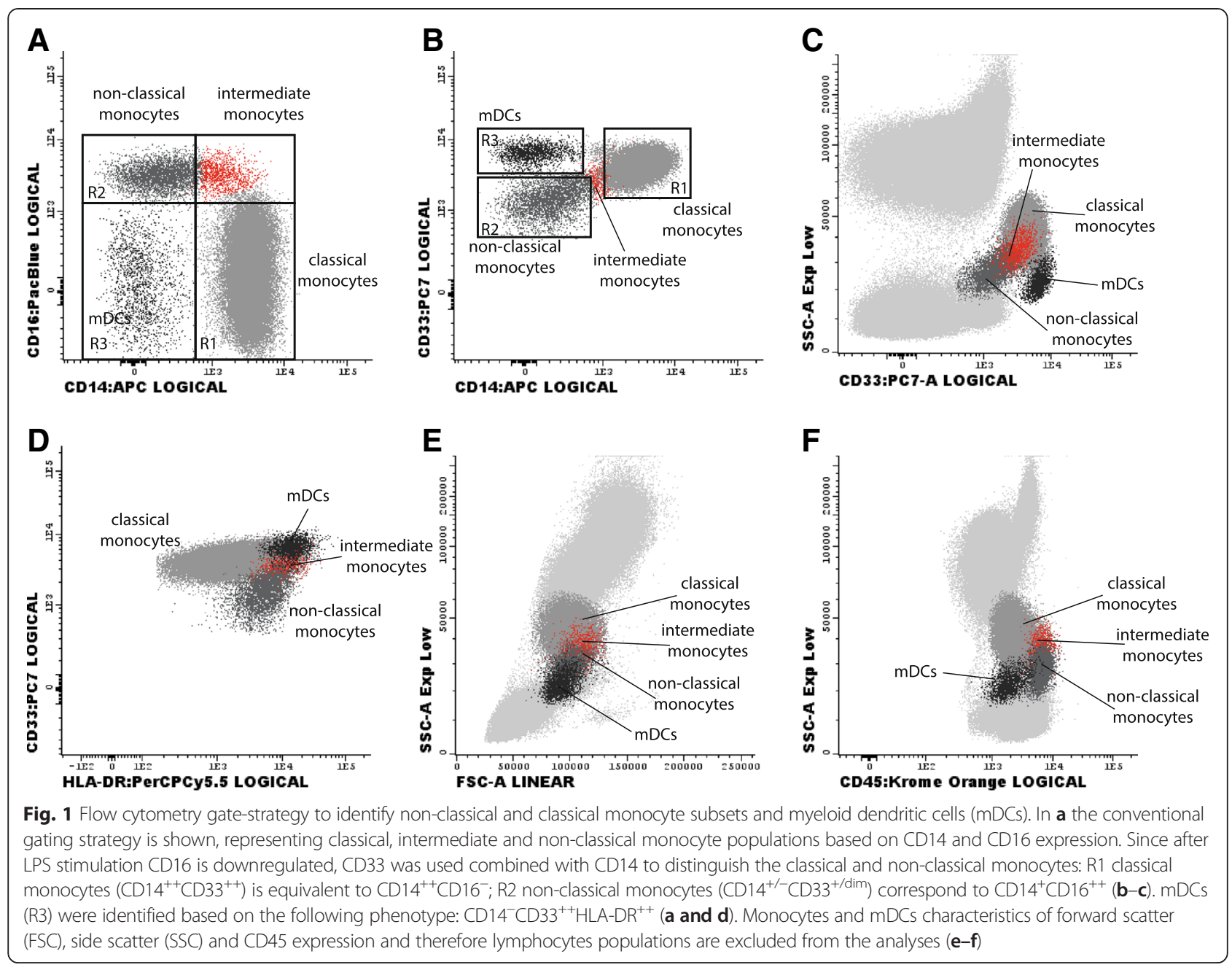

$2 \mathrm{mM}$ L-glutamine and antibiotic-antimycotic agent. $0.05 \mathrm{~mL}$ of serum was added in a final volume of $0.5 \mathrm{~mL}$ : 3 from ASLE, 3 from ISLE and 3 from HC. Each sample was incubated in quadruplicate for $6 \mathrm{~h}$, at $37^{\circ} \mathrm{C}$ in a sterile environment under $5 \% \mathrm{CO}_{2}$.

For the cell sorting of classical monocytes, nonclassical monocytes and mDCs, cells from each sample were resuspended in a final volume of $1 \mathrm{~mL}$ per sample, and lysed with $10 \mathrm{~mL}$ of $\mathrm{NH}_{4} \mathrm{Cl}$ solution (Sigma) to remove the red blood cells. After $20 \mathrm{~min}$ of incubation, samples were centrifuged ( $5 \mathrm{~min}$, at $540 \times \mathrm{g}$ ) and the cell pellet was stained with the following monoclonal antibodies combination: anti-CD45 Krome Orange, anti-HLA-DR fluorescein isothiocyanate (clone: Immu357; Beckman Coulter - Immunotech), anti-CD14 peridin chlorophyll protein - Cyanin 5.5 (clone:M5E2; BD Pharmingen), anti-CD33 phycoerythrin (clone:P67.6; BD Biosciences) and anti-CD123 allophycocyanin (clone: 9 F5; BD Pharmingen). Next, the cells were incubated for $20 \mathrm{~min}$ at room temperature in the dark, washed and resuspended in phosphate-buffered saline (Gibco BRL-life Technologies).

Cell-sorting and purification were performed in FACSAria II cell sorter (BD Biosciences) using the FACSDiva software (BD Biosciences). Classical monocytes were identified and sorted by HLA-DR ${ }^{+} / \mathrm{CD} 14^{\text {high }} / \mathrm{CD} 33^{\text {high }} /$ CD $45^{\text {high }}$ phenotype, non-classical monocytes were HLA$\mathrm{DR}^{\text {inter }} / \mathrm{CD} 33^{\text {inter }} / \mathrm{CD} 123^{\text {neg }} \mathrm{CD} 45^{\text {high }}$, and $\mathrm{mDCs}$ were defined as HLA-DR ${ }^{\text {high }} / \mathrm{CD} 33^{\text {high }} / \mathrm{CD} 14^{\text {neg }} / \mathrm{CD} 123^{\text {neg }}$.

Sorted cell populations were then centrifuged for $5 \mathrm{~min}$ at $300 \times g$ and the pellets were resuspended in $350 \mu \mathrm{L}$ of RLT Lysis Buffer (Qiagen, Hilden, Germany). Total RNA extraction was performed with the RNeasy Micro kit (Qiagen) according to the supplier's instructions. Total RNA was eluted in a $14 \mu \mathrm{l}$ volume of RNase-free water. In order to quantify the amount of total RNA extracted and verify RNA integrity, samples were analyzed using a 6000 Nano Chip kit, in an Agilent 2100 bioanalyzer (Agilent Technologies, Walbronn, Germany) and 2100 expert software, according to the 
manufacturer's instructions. RNA was reverse transcribed with iScript ${ }^{\mathrm{TM}}$ Reverse Transcription Supermix for RTqPCR (Bio-Rad, Hercules, Calif., USA), according to the manufacturer's instructions. Relative quantification of gene expression using real-time PCR was performed in the LightCycler 480 II (Roche Diagnostics, Rotkreuz, Switzerland). Real-time PCR reactions were carried out using $1 \times$ QuantiTect SYBR Green PCR Master Mix (Qiagen), $1 \times$ QuantiTect Primer Assay TRAF1 (QT00095732) (Qiagen) and 20 ng of cDNA sample, in a total volume of $10 \mu \mathrm{L}$. The reactions were performed using the following thermal profile: $15 \mathrm{~min}$ at $95{ }^{\circ} \mathrm{C}$, and 50 cycles of $15 \mathrm{~s}$ at $94{ }^{\circ} \mathrm{C}, 30 \mathrm{~s}$ at $55^{\circ} \mathrm{C}$ and $30 \mathrm{~s}$ at $72{ }^{\circ} \mathrm{C}$. Melting point analysis was done to ensure amplification of the specific product. Real-time PCR results were analyzed with the LightCycler software (Roche Diagnostics). GeNorm Reference Gene Selection kit (PrimerDesign Ltd., Southampton, England) in conjunction with the geNorm software (PrimerDesign Ltd.) were used to select the reference genes to normalize data. The reference genes used for gene expression analysis of classical, non-classical monocytes and $\mathrm{mDC}$ were the beta-2-microglobulin (B2M) (QT00088935) and the ubiquitin-c (UBC) (QT00234430). The normalized TNF- $\alpha$ gene expression were calculated using the delta-Ct method [31].

\section{Serum cytokine quantitation}

Measurements of IL-17F, IL-17A, IL1-7E, IL-10, IL12p70, IL-13, IL-15, IL-22, IL-21, IL-23, IFN- $\gamma$ and TNF- $\alpha$ were performed in all serum samples by Luminex xMAP using the MILLIPLEX MAP Human TH17 Magnetic Bead Panel (EMD Millipore, Billerica, MA, USA). The serum cytokine levels were determined by comparison with a standard curve obtained using the corresponding recombinant human cytokines

\section{Statistical analyses}

Results were expressed as median/mean and range/interquartile range. Statistical evaluation of data was performed through non-parametric tests: The $\chi^{2}$ and Fisher's exact tests were used to evaluate the significance of associations between categorical variables. Continuous variables were compared by Kruskal-Wallis test and Mann-Whitney $U$ test. A Spearman's rank correlation was applied to assess the association between different parameters. The statistical analyses were performed using Statistical Package for Social Sciences IBM SPSS 20 (IBM, Armonk, NY. USA) and Graphpad Prism version 5 (GraphPad Software, San Diego, CA, USA). Differences were considered statistically significant when the $\mathrm{p}$ value was less than 0.05 .

\section{Results}

Higher TNF- $a$ intracellular expression in classical and nonclassical peripheral blood monocytes, after TLR4 ligand stimulation in the presence of sera from SLE patients with active disease

In the absence of LPS, the production of TNF- $\alpha$ by the various monocyte subsets or $\mathrm{mDCs}$ was not significantly affected by the addition of sera from patients with active or inactive SLE as compared to sera from HCs. The frequency of these cells producing TNF- $\alpha$ was consistently below $5 \%$.

In the presence of the TLR4 ligand - LPS, co-culture with sera from patients with ASLE resulted in a higher intracellular expression of TNF- $\alpha$ (MFI) in classical and non-classical monocytes, in comparison to cells cultured with sera from HCs. Sera from patients with ISLE did not reveal differences between ASLE or HC. None of the SLE sera changed the TNF- $\alpha$ expression in $\mathrm{mDCs}$ cotreated with sera from the different groups (Fig. 2a). No differences were observed in the frequency of classical, non-classical monocytes or mDCs expressing TNF- $\alpha$ in the presence of sera from SLE patients when compared to HC sera (Table 2).

Sera of SLE patients with moderate to high positive levels of anti-dsDNA antibodies demonstrated an increased ability to induce TNF-a expression in classical and nonclassical monocytes, co-stimulated with TLR4 ligand SLE patients were categorized according to the serum levels of anti-dsDNA antibodies into negative-low positive (<20 IU) and moderate-high positive (>20 IU) (Table 1). Data analysis demonstrated that classical monocytes exposed to sera from patients with moderate-high positive anti-dsDNA antibodies presented a higher TNF- $\alpha$ expression than those exposed to sera from HC (Fig. 2b). Nonclassical monocytes followed a similar trend but without reaching statistical significance (Fig. 2b). As before, no differences were demonstrated for TNF- $\alpha$ expression in mDCs (Fig. 2b).

High TNF-a levels in the serum of SLE donors is associated with higher intracellular expression of TNF- $a$ in mDCs but not with peripheral blood monocytes

We next investigated the relationship between TNF- $\alpha$ production in monocytes subsets and $\mathrm{mDCs}$ and the levels of IL-17F, IL-17A, IL-17E, IL-10, IL-12p70, IL-13, IL-15, IL-22, IL-21, IL-23, IFN- $\gamma$ and TNF- $\alpha$ in the serum added to the cultures. Our data revealed a positive correlation between the levels of TNF- $\alpha$ in the sera of SLE patients and the intracellular TNF- $\alpha$ expression in mDCs (Fig. 3). These levels were not correlated with intracellular TNF- $\alpha$ expression in peripheral blood monocytes. No significant correlations was found between the levels of TNF- $\alpha$ in the sera from HCs and 


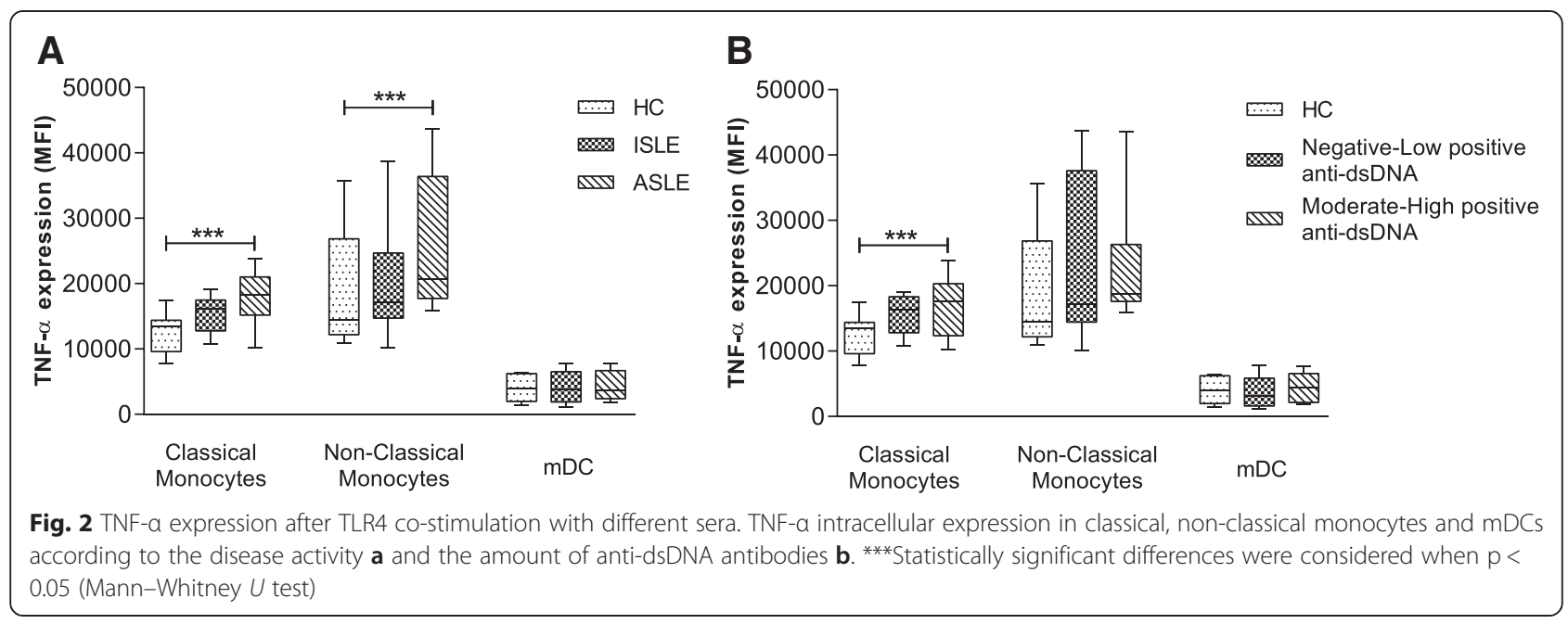

intracellular TNF- $\alpha$ expression in either mDCs $(p=0.535)$ or monocytes subsets.

The others cytokines levels did not correlate with the intracellular expression of TNF- $\alpha$ either in cultured mDCs or monocyte subsets. Additionally, no differences were found in the cytokines levels between the three groups of participants (Table 3 ).

\section{TNF-a mRNA gene expression in sorted monocytes} subsets and $\mathrm{mDCs}$ in the presence of SLE patient's serum without LPS co-stimulation

Since the presence of SLE sera alone did not demonstrated the ability to shift the TNF- $\alpha$ intracellular expression in monocytes subsets and mDCs as measured by flow cytometry, we next used qRT-PCR to measure the TNF- $\alpha$ mRNA expression in these purified cells after treatment with SLE or HC sera, in the absence of LPS. For this analysis, we selected 6 SLE sera (3 ASLE patients and 3 ISLE patients) and $3 \mathrm{HC}$ sera. Only a trend for a higher TNF- $\alpha$ mRNA expression in the presence of SLE sera versus HC sera in both classical and nonclassical monocytes was observed (Fig. 4), although no statically significant differences were observed.

\section{Discussion}

SLE is a chronic autoimmune disorder associated with a large number of immunological abnormalities, which includes phenotypic and functional alterations in monocytes and DCs [14, 15, 32, 33]. These cell types have an increasingly recognized role in this complex disease. Soluble mediators present in SLE patient's sera are able to perpetuate the cellular activation in this disease $[20,34,35]$, however their effects on monocyte subsets (classical and non-classical monocytes), as well as mDCs are poorly understood.

The production of pro-inflammatory cytokines, such as TNF- $\alpha$, can be directly stimulated by signaling immunoglobulin receptors, complement receptors or through several other cellular receptors, including pattern recognition receptors as TLRs [36]. The TNF- $\alpha$ intracellular expression in classical, non-classical monocytes and mDCs remained unchanged when these cells are exposed to sera form ISLE, ASLE or HCs. This may be due to the absence of sufficient amounts of primary TNF- $\alpha$ production activators in these sera. However, it is also possible that the expression of inhibitory Fc gamma receptors $(\mathrm{Fc} \gamma \mathrm{R})$, particularly FcyRIIB, is stimulated in these cells which may bind IgG immune complexes without triggering activation but preventing self-responses [37].

On other hand, in the presence of TLR4 ligand, classical and non-classical monocytes co-treated with sera from ALSE exhibit a higher production of TNF- $\alpha$ when compared to cells exposed to sera from HCs. Our data suggests that TLR4 activation in the presence of ASLE

Table 2 Frequency of classical, non-classical monocytes and mDCs producing TNF-a

\begin{tabular}{llll}
\hline & Classical monocytes & Non-classical monocytes & mDCs \\
\hline Basal & $2.08(1.27-3.19)$ & $4.78(3.99-5.12)$ & $5.67(2.94-7.79)$ \\
TLR4 ligand & $95.81(93.92-96.46)$ & $84.67(49.64-99.323)$ & $65.66(61.98-78.30)$ \\
HC sera + TLR4 ligand & $95.80(93.40-97.55)$ & $95.69(82.25-99.66)$ & $64.04(35.68-77.44)$ \\
ISLE sera + TLR4 ligand & $95.59(93.88-98.51)$ & $96.02(81.13-98.98)$ & $42.51(26.03-77.20)$ \\
ASLE sera + TLR4 ligand & $97.01(92.53-98.96)$ & $93.33(72.85-99.35)$ & $41.03(26.35-72.9)$ \\
\hline
\end{tabular}

Results expressed in percentage (\%) as median (minimum-maximum) 

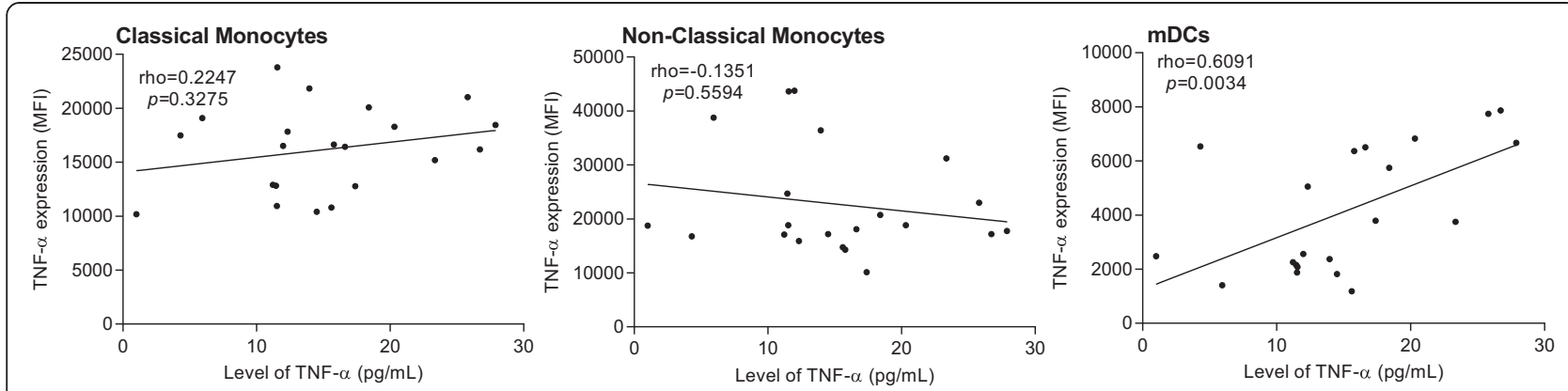

Fig. 3 Correlation between SLE sera TNF-a levels and TNF-a intracellular expression after TLR4 co-stimulation with different sera. Statistical significant differences were considered when $p<0.05$. The correlations were assessed by the Spearman's rank correlation

sera results in an aberrant response by classical and non-classical monocytes. These results are in line with the findings of Leadbetter et al. that reported an aberrant B-cell response mediated by IgG complexes and TLR4 activation in their mice experiments [38].

In order to better define which serum components could be implicated in the activation of monocytes, we explored the relationship between this activations and the serum levels of anti-dsDNA antibodies and cytokines. Remarkably, moderate-high levels of anti-dsDNA were associated with more intense activation of TNF- $\alpha$ production by classical and non-classical monocytes. This finding enhances the hypothesis that the presence of auto-antibodies and immune-complexes, with the

Table 3 Cytokine levels obtained in the 22 SLE patients and 10 healthy individuals

\begin{tabular}{|c|c|c|c|c|c|}
\hline & & $\mathrm{HC} n=10$ & ISLE $n=11$ & ASLE $n=11$ & $P$ value significance \\
\hline \multirow[t]{2}{*}{ IL-17A } & Cytokine level (pg/mL) & $0.0(0.0-0.0): 0.00$ & 0.0 (0.0-113.40): 12.69 & 0.0 (0.0-62.04): 9.28 & ns \\
\hline & $\%$ of samples detected (n) & $0 \%(n=0)$ & $18.2 \%(n=2)$ & $27.3 \%(n=3)$ & ns \\
\hline \multirow[t]{2}{*}{ IL-17E } & Cytokine level (pg/mL) & $0.0(0.0-286.21): 81.27$ & 0.0 (0.0-1364.68): 159.38 & 0.0 (0.0-57.10): 5.19 & ns \\
\hline & $\%$ of samples detected (n) & $30 \%(n=3)$ & $18.2 \%(n=2)$ & $9.1 \%(n=1)$ & ns \\
\hline \multirow[t]{2}{*}{ IL-17F } & Cytokine level (pg/mL) & 0.0 (0.0-14.49): 3.93 & 0.0 (0.00-158.44): 19.10 & 0.0 (0.0-57.10): 5.19 & ns \\
\hline & $\%$ of samples detected $(n)$ & $30 \%(n=3)$ & $27.3 \%(n=3)$ & $9.1 \%(n=1)$ & ns \\
\hline \multirow[t]{2}{*}{ IL-12p70 } & Cytokine level (pg/mL) & 0.0 (0.0-46.87): 6.98 & 0.96 (0.0-60.76): 8.05 & $0.0(0.0-85.52): 8.85$ & ns \\
\hline & $\%$ of samples detected (n) & $30 \%(n=3)$ & $54.5 \%(n=6)$ & $36.4 \%(n=4)$ & ns \\
\hline \multirow[t]{2}{*}{ IL-23 } & Cytokine level (pg/mL) & 0.0 (0.0-1134.54): 276.46 & 0.0 (0.0-11221.86): 1216.17 & 0.0 (0.0-2547.96): 258.94 & ns \\
\hline & $\%$ of samples detected (n) & $30 \%(n=3)$ & $18.2 \%(n=2)$ & $18.2 \%(n=2)$ & ns \\
\hline \multirow[t]{2}{*}{ TNF-a } & Cytokine level (pg/mL) & 7.68 (6.25-33.50): 12.38 & 13.57 (4.30-26.73): 13.66 & 14.51 (1.01-27.90): 16.47 & ns \\
\hline & $\%$ of samples detected (n) & $100 \%(n=10)$ & $100 \%(n=11)$ & $100 \%(n=11)$ & ns \\
\hline \multirow[t]{2}{*}{$\mathrm{IFN}-\gamma$} & Cytokine level (pg/mL) & 0.0 (0.0-8.96): 2.95 & $0.0(0.0-167.11): 21.90$ & $2.77(0.0-79.40): 12.36$ & ns \\
\hline & $\%$ of samples detected (n) & $40 \%(n=4)$ & $27.3 \%(n=3)$ & $63.6 \%(n=7)$ & ns \\
\hline \multirow[t]{2}{*}{$\mathrm{IL}-15$} & Cytokine level (pg/mL) & $0.0(0.0-0.0): 0.0$ & $0.0(0.0-7.45): 1.41$ & $0.0(0.0-0.0): 0.0$ & ns \\
\hline & $\%$ of samples detected (n) & $0 \%(n=0)$ & $18.2 \%(n=2)$ & $0 \%(n=0)$ & ns \\
\hline \multirow[t]{2}{*}{$\mid \mathrm{L}-10$} & Cytokine level (pg/mL) & $0.0(0.0-0.0): 0.0$ & 0.0 (0.0-6.58): 0.99 & 0.0 (0.0-5.36): 0.89 & ns \\
\hline & $\%$ of samples detected (n) & $0 \%(n=0)$ & $100 \%(n=11)$ & $100 \%(n=11)$ & ns \\
\hline \multirow[t]{2}{*}{ IL-22 } & Cytokine level (pg/mL) & 0.0 (0.0-100.8): 18.77 & 0.0 (0.0-598.67): 64.06 & $0.0(0.0-159.85): 14.53$ & ns \\
\hline & $\%$ of samples detected $(n)$ & $30 \%(n=3)$ & $18.2 \%(n=2)$ & $9.1 \%(n=1)$ & ns \\
\hline \multirow[t]{2}{*}{$\| \mathrm{L}-21$} & Cytokine level (pg/mL) & $0.0(0.0-5.61): 0.80$ & 0.0 (0.0-105.58): 12.13 & $0.0(0.0-24.07): 2.68$ & ns \\
\hline & $\%$ of samples detected (n) & $10 \%(n=1)$ & $18.2 \%(n=2)$ & $18.2 \%(n=2)$ & ns \\
\hline \multirow[t]{2}{*}{$\mathrm{IL}-13$} & Cytokine level (pg/mL) & $0.0(0.0-0.0): 0.0$ & 0.0 (0.0-56.44): 6.76 & $0.0(0.0-0.0): 0.0$ & ns \\
\hline & $\%$ of samples detected $(n)$ & $0 \%(n=0)$ & $18.8 \%(n=2)$ & $0 \%(n=0)$ & ns \\
\hline
\end{tabular}

Results expressed in $\mathrm{pg} / \mathrm{mL}$ as median (minimum-maximum): mean

$\%$ and $(\mathrm{n}=)$ represent the percentage (number of cases) of samples detected

$p$ values were calculated by Kruskal-Wallis test and Mann-Whitney $U$ test for continuous variables, and $x^{2}$ and Fisher's exact tests were used to measure associations between categorical variables. ns: non-significant 

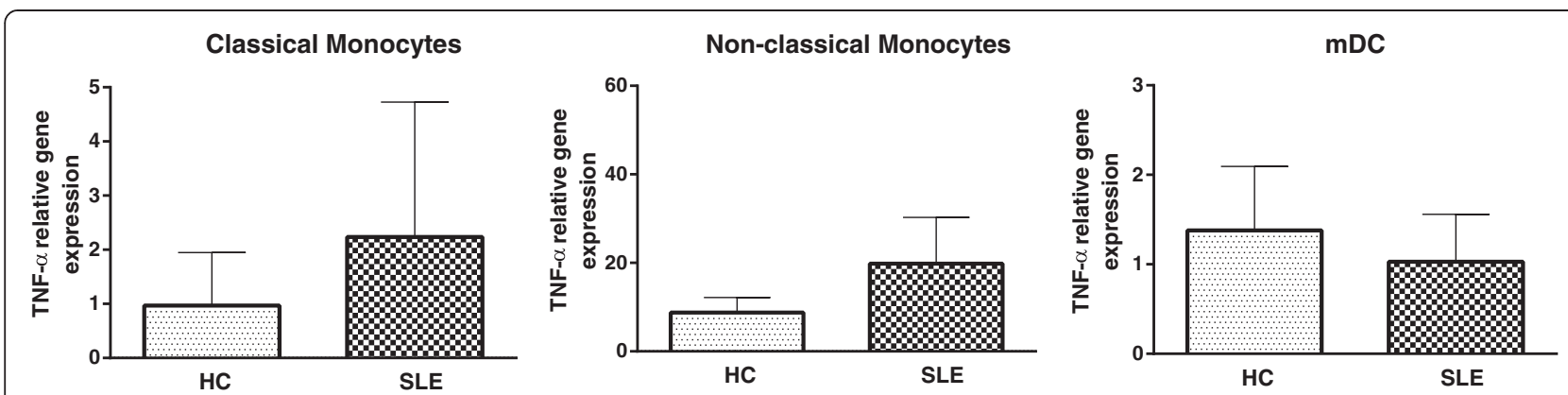

Fig. 4 TNF-a relative gene expression in sorted classical, non-classical monocytes and mDCs stimulated with sera. Results expressed as mean and standard deviation

ability to activate Fc $\gamma \mathrm{R}$ and TLRs, leads to increased production of TNF- $\alpha[20,39]$. This is supported by the evidence that cross-linking between IgGs to Fc $\gamma$ Rs triggers a wide variety of cellular functions, including release of inflammatory mediators, like cytokines, chemokines and reactive oxygen species $[40,41]$.

mDCs was not sensitive to the effects of sera of these diverse origins upon TNF- $\alpha$ expression, both in the absence and in the presence of LPS. This suggests that this subpopulation of DCs is less sensitive to a peripheral inflammatory environment, probably due to the fact that the majority of $\mathrm{PB} \mathrm{mDCs}$ have an immature phenotype $[14,15]$. In fact, the different pattern of TNF- $\alpha$ expression observed in classical monocytes, non-classical monocytes and $\mathrm{mDCs}$ can also be related with the differential expression of FcyRs. Classical monocytes constitutively express CD64 (Fc $\gamma \mathrm{RI})$, a high affinity receptor, while non-classical monocytes expresses CD16 (FcyRIII), a low affinity receptor, and mDCs express low levels of CD32 (FcyRII) an intermediate affinity receptor [42-44].

We also explored the relationship between TNF- $\alpha$ expression and the levels of a large array of cytokines in the serum added to the cultures. Interestingly, we observed a positive correlation between TNF- $\alpha$ sera levels and TNF- $\alpha$ intracellular expression in $\mathrm{mDC}$ co-stimulated with TLR4 ligand in the presence of SLE sera. This finding is in line with the ability of soluble TNF- $\alpha$ induces mDCs maturation as well as TNF- $\alpha$ production $[45,46]$.

Finally, we analyzed whether SLE sera in the absence of TLR4 ligand, could result in alterations of TNF- $\alpha$ mRNA expression in classical, non-classical monocytes and mDCs. A slight, non-significant increase in the TNF- $\alpha$ mRNA expression was observed in both monocyte subsets in the presence of SLE sera.

\section{Conclusion}

In summary, our data demonstrated that sera from patients with active SLE increase TNF- $\alpha$ production by classical and non-classical monocytes, in the presence of
LPS. This effect may be partially explained by circulating auto-antibodies since the high levels of anti-dsDNA antibodies are associated with an enhanced TNF- $\alpha$ intracellular expression. This supports the concept that the presence of the immune-complexes is an important factor in cell activation and in the maintenance of chronic inflammation in SLE.

\section{Abbreviations}

ASLE: Systemic lupus erythematosus patients in active disease; DC: Dendritic cell; dsDNA: Double stranded DNA; FcyR: Fc gamma receptor; IL: Interleukin; IFN: Interferon; ISLE: Systemic lupus erythematosus patients in inactive disease; LPS: Lipopolysaccharide; mDC: Myeloid dendritic cell; SLE: Systemic lupus erythematosus; SLEDAl: Systemic lupus erythematosus disease activity index 2000; TLR: Toll-like receptor; TNF-a: Tumor necrosis factor a.

\section{Competing interests}

The authors declare that they have no competing interests.

\section{Authors' contributions}

TC carried the analysis and interpretation of data, the statistical analysis and drafted the manuscript; DM performed cell cultures and flow cytometry assays; LAP and HAY determined the sera cytokines levels; LI and JAPS provided patients samples and their clinical information; AL and AM participated in the molecular studies; AH has been involved in manuscript revising; SP performed the cell sorting; HT, JAPS and AP contributed to conception and designed the study protocol and given final approval of the version to be published. All authors read and approved the final manuscript.

\section{Acknowledgments}

This work was partially supported by research grant from the Portuguese Society of Rheumatology (SPR): "Bolsa de Investigação na área das Doenças Reumáticas Inflamatórias - Bolsa SPR/MSD 2014" and by the Intramural Research Program of the NIH, National Cancer Institute.

\section{Author details}

${ }^{1}$ Blood and Transplantation Center of Coimbra, Portuguese Institute of Blood and Transplantation, Quinta da Vinha Moura, São Martinho do Bispo, 3041-861 Coimbra, Portugal. ${ }^{2}$ College of Health Technology of Coimbra, Rua 5 de Outubro, São Martinho do Bispo, 3046-854 Coimbra, Portugal. ${ }^{3} \mathrm{HPV}$ Immunology Laboratory, Frederick National Laboratory for Cancer Research, Building 469, 21702 Frederick, MD, USA. ${ }^{4}$ Rheumatology Department, Centro Hospitalar e Universitário de Coimbra, Praceta Prof. Mota Pinto, 3000-075 Coimbra, Portugal. ${ }^{5}$ Faculty of Medicine, University of Coimbra, Azinhaga de Santa Comba, Celas, 3000-548 Coimbra, Portugal. 'S School of Health Sciences, University of Beira Interior, Avenida Infante D. Henrique, 6200-506 Covilhã, Portugal. 'Laboratory of Experimental Immunology, Cancer and Inflammation Program, National Cancer Institute at Frederick, Building 560, 21702-1201 Frederick, MD, USA. 
Received: 11 January 2015 Accepted: 18 May 2015

Published online: 23 May 2015

\section{References}

1. Agmon-Levin N, Mosca M, Petri M, Shoenfeld Y. Systemic lupus erythematosus one disease or many? Autoimmun Rev. 2012;11:593-5.

2. Fu SM, Deshmukh US, Gaskin F. Pathogenesis of systemic lupus erythematosus revisited 2011: end organ resistance to damage, autoantibody initiation and diversification, and HLA-DR. J Autoimmun. 2011;37:104-12.

3. Mok CC, Lau CS. Pathogenesis of systemic lupus erythematosus. J Clin Pathol. 2003;56:481-90.

4. O'Neill S, Cervera R. Systemic lupus erythematosus. Best Pract Res Clin Rheumatol. 2010;24:841-55.

5. Marks SD, Tullus K. Autoantibodies in systemic lupus erythematosus. Pediatr Nephrol. 2012;27:1855-68.

6. Wong KL, Yeap WH, Tai JJ, Ong SM, Dang TM, Wong SC. The three human monocyte subsets: implications for health and disease. Immunol Res. 2012:53:41-57.

7. Auffray C, Sieweke MH, Geissmann F. Blood monocytes: development, heterogeneity, and relationship with dendritic cells. Annu Rev Immunol. 2009:27:669-92.

8. Ziegler-Heitbrock L, Ancuta P, Crowe S, Dalod M, Grau V, Hart DN, et al. Nomenclature of monocytes and dendritic cells in blood. Blood. 2010;116:e74-80.

9. Mosig S, Rennert K, Krause S, Kzhyshkowska J, Neunubel K, Heller R, et al. Different functions of monocyte subsets in familial hypercholesterolemia: potential function of CD14+ CD16+ monocytes in detoxification of oxidized LDL. FASEB J. 2009;23:866-74.

10. Soehnlein $\mathrm{O}$, Lindbom L. Phagocyte partnership during the onset and resolution of inflammation. Nat Rev Immunol. 2010;10:427-39.

11. Cros J, Cagnard N, Woollard K, Patey N, Zhang SY, Senechal B, et al. Human CD14dim monocytes patrol and sense nucleic acids and viruses via TLR7 and TLR8 receptors. Immunity. 2010;33:375-86.

12. Pereira Ml, Paiva A. Dendritic cells in cord blood transplantation: a review. Stem Cells Int. 2011;2011:539896.

13. Crespo I, Paiva A, Couceiro A, Pimentel P, Orfao A, Regateiro F. Immunophenotypic and functional characterization of cord blood dendritic cells. Stem Cells Dev. 2004;13:63-70.

14. Carvalheiro T, Rodrigues A, Lopes A, Ines L, Velada I, Ribeiro A, et al. Tolerogenic versus inflammatory activity of peripheral blood monocytes and dendritic cells subpopulations in systemic lupus erythematosus. Clin Dev Immunol. 2012;2012:934161.

15. Henriques A, Ines L, Carvalheiro T, Couto M, Andrade A, Pedreiro S, et al. Functional characterization of peripheral blood dendritic cells and monocytes in systemic lupus erythematosus. Rheumatol Int. 2012;32:863-9.

16. Sule S, Rosen A, Petri M, Akhter E, Andrade F. Abnormal production of pro- and anti-inflammatory cytokines by lupus monocytes in response to apoptotic cells. PLoS One. 2011;6:e17495.

17. Crispin JC, Alcocer-Varela J. The role myeloid dendritic cells play in the pathogenesis of systemic lupus erythematosus. Autoimmun Rev. 2007:6:450-6.

18. Fransen JH, van der Vlag J, Ruben J, Adema GJ, Berden JH, Hilbrands LB. The role of dendritic cells in the pathogenesis of systemic lupus erythematosus. Arthritis Res Ther. 2010;12:207.

19. Chiang EY, Yu X, Grogan JL. Immune complex-mediated cell activation from systemic lupus erythematosus and rheumatoid arthritis patients elaborate different requirements for IRAK1/4 kinase activity across human cell types. J Immunol. 2011;186:1279-88.

20. Means TK, Latz E, Hayashi F, Murali MR, Golenbock DT, Luster AD. Human lupus autoantibody-DNA complexes activate DCs through cooperation of CD32 and TLR9. J Clin Invest. 2005;115:407-17.

21. Blanco P, Palucka AK, Gill M, Pascual V, Banchereau J. Induction of dendritic cell differentiation by IFN-alpha in systemic lupus erythematosus. Science. 2001;294:1540-3.

22. Joo H, Coquery C, Xue Y, Gayet I, Dillon SR, Punaro M, et al. Serum from patients with SLE instructs monocytes to promote lgG and IgA plasmablast differentiation. J Exp Med. 2012;209:1335-48,

23. Bombardier C, Gladman DD, Urowitz MB, Caron D, Chang CH. Derivation of the SLEDAI. A disease activity index for lupus patients. The Committee on prognosis studies in SLE. Arthritis Rheum. 1992:35:630-40.
24. Gladman DD, Ibanez D, Urowitz MB. Systemic lupus erythematosus disease activity index 2000. J Rheumatol. 2002;29:288-91.

25. Griffiths B, Mosca M, Gordon C. Assessment of patients with systemic lupus erythematosus and the use of lupus disease activity indices. Best Pract Res Clin Rheumatol. 2005;19:685-708.

26. Kalina T, Flores-Montero J, van der Velden VH, Martin-Ayuso M, Bottcher S, Ritgen $M$, et al. EuroFlow standardization of flow cytometer instrument settings and immunophenotyping protocols. Leukemia. 2012;26:1986-2010.

27. Belge KU, Dayyani F, Horelt A, Siedlar M, Frankenberger M, Frankenberger B, et al. The proinflammatory CD14 (+) CD16 (+) DR (++) monocytes are a major source of TNF. J Immunol. 2002;168:3536-42.

28. Dimitrov S, Shaikh F, Pruitt C, Green M, Wilson K, Beg N, et al. Differential TNF production by monocyte subsets under physical stress: blunted mobilization of proinflammatory monocytes in prehypertensive individuals. Brain Behav Immun. 2013;27:101-8.

29. Picozza M, Battistini L, Borsellino G. Mononuclear phagocytes and marker modulation: when CD16 disappears, CD38 takes the stage. Blood. 2013;122:456-7.

30. Poehlmann H, Schefold JC, Zuckermann-Becker H, Volk HD, Meisel C. Phenotype changes and impaired function of dendritic cell subsets in patients with sepsis: a prospective observational analysis. Crit Care. 2009;13:R119.

31. Vandesompele J, De Preter K, Pattyn F, Poppe B, Van Roy N, De Paepe A, et al. Accurate normalization of real-time quantitative RT-PCR data by geometric averaging of multiple internal control genes. Genome Biol. 2002;3:RESEARCH0034.

32. Gerl V, Lischka A, Panne D, Grossmann P, Berthold R, Hoyer BF, et al. Blood dendritic cells in systemic lupus erythematosus exhibit altered activation state and chemokine receptor function. Ann Rheum Dis. 2010;69:1370-7.

33. Li Y, Lee PY, Reeves WH. Monocyte and macrophage abnormalities in systemic lupus erythematosus. Arch Immunol Ther Exp (Warsz). 2010;58:355-64.

34. Sato Y, Miyata M, Sato Y, Nishimaki T, Kochi H, Kasukawa R. CpG motif-containing DNA fragments from sera of patients with systemic lupus erythematosus proliferate mononuclear cells in vitro. J Rheumatol. 1999;26:294-301.

35. Ronnelid J, Tejde A, Mathsson L, Nilsson-Ekdahl K, Nilsson B. Immune complexes from SLE sera induce IL10 production from normal peripheral blood mononuclear cells by an FcgammaRIl dependent mechanism: implications for a possible vicious cycle maintaining B cell hyperactivity in SLE. Ann Rheum Dis. 2003;62:37-42.

36. Lacy P, Stow JL. Cytokine release from innate immune cells: association with diverse membrane trafficking pathways. Blood. 2011;118:9-18.

37. Ravetch JV, Bolland S. IgG Fc receptors. Annu Rev Immunol. 2001;19:275-90.

38. Leadbetter EA, Rifkin IR, Hohlbaum AM, Beaudette BC, Shlomchik MJ, MarshakRothstein A. Chromatin-lgG complexes activate B cells by dual engagement of IgM and Toll-like receptors. Nature. 2002;416:603-7.

39. Nielsen $\mathrm{CT}$, Ostergaard O, Johnsen C, Jacobsen S, Heegaard NH. Distinct features of circulating microparticles and their relationship to clinical manifestations in systemic lupus erythematosus. Arthritis Rheum. 2011;63:3067-77.

40. Gessner JE, Heiken H, Tamm A, Schmidt RE. The IgG Fc receptor family. Ann Hematol. 1998;76:231-48.

41. Nielsen CT, Ostergaard O, Stener L, Iversen LV, Truedsson L, Gullstrand B, et al. Increased lgG on cell-derived plasma microparticles in systemic lupus erythematosus is associated with autoantibodies and complement activation. Arthritis Rheum. 2012;64:1227-36.

42. Bruhns P. Properties of mouse and human lgG receptors and their contribution to disease models. Blood. 2012;119:5640-9.

43. Almeida J, Bueno C, Alquero MC, Sanchez ML, Canizo MC, Fernandez ME, et al. Extensive characterization of the immunophenotype and pattern of cytokine production by distinct subpopulations of normal human peripheral blood MHC II+/lineage- cells. Clin Exp Immunol. 1999;118:392-401.

44. Almeida J, Bueno C, Alguero MC, Sanchez ML, de Santiago M, Escribano L, et al. Comparative analysis of the morphological, cytochemical, immunophenotypical, and functional characteristics of normal human peripheral blood lineage (-)/CD16(+)/HLA-DR(+)/CD14(-/lo) cells, CD14 (+) monocytes, and CD16 (-) dendritic cells. Clin Immunol. 2001;100:325-38.

45. Banchereau J, Steinman RM. Dendritic cells and the control of immunity. Nature. 1998:392:245-52.

46. Nelson EL, Strobl S, Subleski J, Prieto D, Kopp WC, Nelson PJ. Cycling of human dendritic cell effector phenotypes in response to TNF-alpha: modification of the current 'maturation' paradigm and implications for in vivo immunoregulation. FASEB J. 1999;13:2021-30. 